\title{
Phenomenological Percolation in Conceptual Networks associated to Complex Systems
}

\author{
Gonzalo A. Aranda-Corral \\ Department of Information Technology \\ Universidad de Huelva \\ Palos de La Frontera, Spain
}

\author{
Joaquín Borrego-Díaz \\ Dept. Computer Science and AI \\ Universidad de Sevilla \\ Sevilla, Spain
}

\author{
Juan Galán-Páez(*) \\ Dept. Computer Science and AI \\ Universidad de Sevilla \\ Sevilla, Spain
}

\begin{abstract}
A notion of phenomenological percolation for Conceptual Structures associated to Complex Systems (CS) is proposed. This process on concept lattices (from Formal Concept Analysis) consists in the allocation of (non-previous) objects in the lattice, which can induce its extension. The aim is to study the robustness of this conceptual structure when facing unexpected changes (represented by the new object) in the CS it models. Results show that concept lattices associated to CS present more robustness than concept lattices modeling other kind of systems.
\end{abstract}

\section{INTRODUCTION}

A persistent challenge in Complex Systems (CS) research is the phenomenological reconstruction of systems from raw data [14]. In order to face the problem, the use of sound features to reason on the system is a key step. The task of understanding a phenomenon aims to find a reasonably precise and concise approximation to this phenomenon and its behavior such that it can be grasped by the human brain.

New methods and tools have to be developed in order to assist experimental design and interpretation for: Identifying relevant entities at a given time and space scale, characterizing interactions between entities, and finally assessing and formalizing the system behavior [9]. For example, Physics investigation is based on building models of reality: in order to understand a phenomenon, we need to represent it in our minds using a limited amount of symbols. However, it is a common experience that, even using simple "building blocks" it is possible to obtain systems whose behavior is quite complex. In this case it is necessary to develop new languages and new phenomenological models in order to manage this "complexity" [5]. Moreover, it is highly possible that a characterization expires due to the CS evolution and, as a consequence of this, the emergence of not previously defined concepts.

Therefore it is necessary to evaluate how the concept structure describing the CS evolves. Particularly interesting is the case in which non-classifiable (unexpected) objects (events) appear, as it implies the reparation of the conceptual structure. From a cognitive point of view, the conceptual structure -in the case of data from observations- comes from situated conceptualizations: When a situation is experienced repeatedly, multimodal knowledge accrues in the respective simulators for the relevant people, objects, actions, introspections, and settings. The conceptualization's components become entrenched in the respective simulators, as do associations between these components. Over time, the situated conceptualization becomes so well established that it comes to mind automatically and immediately as a unity when the situation arises [6]. Unexpected situations (observations) have to be conciliated with the pre-existent conceptual system.

In general terms, the representation by means of qualitative models can be slanted to predict certain future states of the CS because they are based on past information. The knowledge retrieved from past observations may not gather information on unprecedented states. Therefore, the model will not be useful if the CS achieves these states. Nevertheless, when the model is based on a (big) certain amount of qualitative information, it is expected that only few changes are necessary to fairly represent the new states. This question is important, for example, to evaluate the soundness of emergent semantic networks representing language dynamics [1] (which is an example of how agent concept acquisition lie at the heart of generalized information, [15]) and also to test sentiment lexicons to represent opinion streaming in social media [7], [8].

Studying the robustness of the system to changes means to study how many of the possible states of the system (assuming they were finite) can be represented in the current model. An interesting way to collect and analyze the available information on the system (previously transformed into qualitative knowledge) is by using formal contexts.

Formal Concept Analysis (FCA) [11] provides a number of mathematical tools useful to build semantic structures (concept lattice, CL) from observable information on a CS. In the case of its application to CS, the concept lattice represents a complex semantic network with interesting topological properties [4]. FCA also provides an associated Knowledge Basis $(\mathrm{KB})$ that is useful for (qualitative) reasoning with observable features. In [3] its application for predicting CS evolution is shown, and in [2] a FCA-based framework for cellular automata is presented.

Aim of the paper The present paper addresses the problem of concept lattice refinement when new observations do not fit with the semantic classification provided by the concept lattice. The problem can be viewed as a percolation problem in 


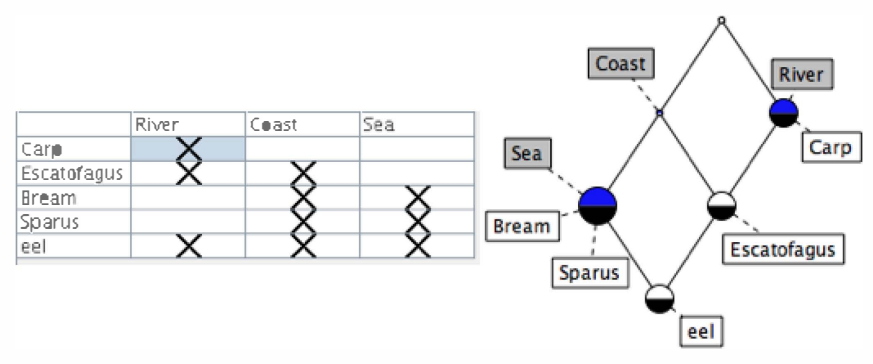

Fig. 1. Formal context of fishes, and its concept lattice

a (semantic) network (phenomenological percolation): Given a new object (event), is it soundly classified by the concept lattice? It seems natural to think that in well-constructed representations of qualitative features of the CS, the concept lattice should allow this classification without any change.

Several types of percolation can be considered: purely classification oriented (that its, how the new element is classified by the CL, to estimate when new concepts should be inserted in the lattice, without applying reasoning), deductive classification (as the former but after inferring new properties on the object prior its classification) and other hybrid methods. In this paper we focus on deductive classification. Experiments show that successful semantic representations present good percolation resistance, while poor CL (from poor representations) or CL non associated to complex systems do not.

\section{BACKGROUND: FORMAL CONCEPT ANALYSIS}

FCA mathematizes the philosophical understanding of a concept as a unit of thoughts composed of two parts: the extent and the intent. The extent covers all objects belonging to the concept, while the intent comprises all common attributes valid for all the objects under consideration [11]. It also allows the computation of concept hierarchies from data tables.

A formal context $M=(O, A, I)$ consists of two sets, $O$ (objects) and $A$ (attributes), and a relation $I \subseteq O \times A$. Finite contexts can be represented by a 1-0-table (identifying $I$ with a boolean function on $O \times A$ ). Given $X \subseteq O$ and $Y \subseteq A$, it defines $X^{\prime}=\{a \in A \mid o I a$ for all $o \in X\}$ and $Y^{\prime}=\{o \in$ $O \mid o I a$ for all $a \in Y\}$

The main goal of FCA is the computation of the concept lattice associated with the context. A (formal) concept is a pair $(X, Y)$ such that $X^{\prime}=Y$ and $Y^{\prime}=X$. For example, the concept lattice from the formal context of fishes of Fig. 1, left (attributes are understood as "live in") is depicted in Fig. 1, right. Each node is a concept, and its intension (or extension) can be formed by the set of attributes (or objects) included along the path to the top (or bottom). For example, the bottom concept (\{eel $\},\{$ Coast, Sea, River $\})$ is the concept euryhaline fish. CL contains every concept that can be extracted from the context. Even if those are defined, it is possible that no specific term (word) exists to denote them.

Logical expressions in FCA are implications between attributes. An implication is a pair of sets of attributes, written
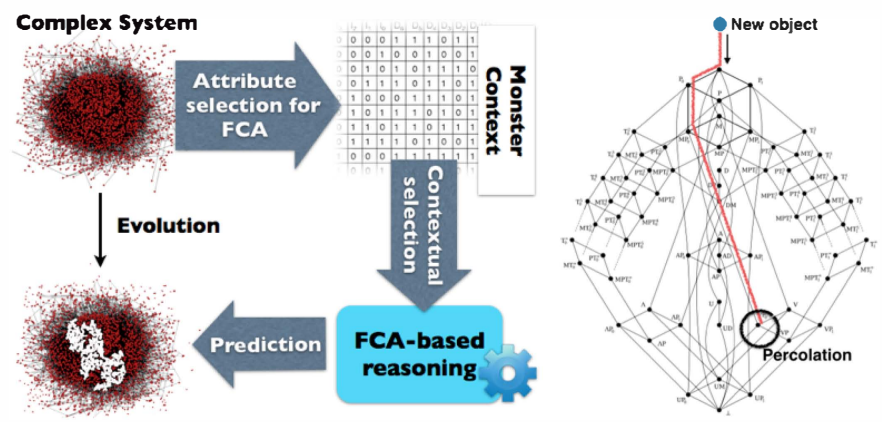

Fig. 2. FCA-based model for qualitative reasoning with Complex Systems (left) and percolation process in Concept Lattices (right)

as $Y_{1} \rightarrow Y_{2}$, which is true with respect to $M=(O, A, I)$ according to the following definition.

A subset $T \subseteq A$ respects $Y_{1} \rightarrow Y_{2}$ if $Y_{1} \nsubseteq T$ or $Y_{2} \subseteq T$. It says that $Y_{1} \rightarrow Y_{2}$ holds in $M\left(M \models Y_{1} \rightarrow Y_{2}\right)$ if for all $o \in O$, the set $\{o\}^{\prime}$ respects $Y_{1} \rightarrow Y_{2}$. In that case, it is said that $Y_{1} \rightarrow Y_{2}$ is an implication of $M$.

Definition. 2.1: Let $\mathcal{L}$ be a set of implications and $L$ be an implication.

1) $L$ follows from $\mathcal{L}(\mathcal{L} \models L)$ if each subset of $A$ respecting $\mathcal{L}$ also respects $L$.

2) $\mathcal{L}$ is complete if every implication of the context follows from $\mathcal{L}$.

3) $\mathcal{L}$ is non-redundant if for each $L \in \mathcal{L}, \mathcal{L} \backslash\{L\} \not \forall L$.

4) $\mathcal{L}$ is a (implication) basis for $M$ if $\mathcal{L}$ is complete and non-redundant.

A basis called Stem Basis (SB)[12] can be obtained from the pseudo-intents. It is important to remark that SB is only an example of a basis for a formal context. In this paper no specific property of the SB is used, so it can be replaced by any implication basis.

The reasoning module is a production system that initially it works with SB, and entailment is based on the following result:

Theorem. 2.2: Let $\mathcal{S}$ be a basis for $M$ and $\left\{A_{1}, \ldots, A_{n}\right\} \cup$ $Y \subseteq A$. The following conditions are equivalent:

1) $\mathcal{S} \cup\left\{A_{1}, \ldots A_{n}\right\} \vdash_{p} Y\left(\vdash_{p}\right.$ is the entailment with respect to a production system).

2) $M \mid=\left\{A_{1}, \ldots A_{n}\right\} \rightarrow Y$.

\section{FCA-BASED REASONING ON CS}

Complex networks are a widely used representation of selected features from a CS. The topological structure of this network aids understanding characteristics of the associated CS. When the goal is to reason with qualitative features, it can be interesting to extract emergent concepts from these interactions [3].

It is here where FCA can play a relevant role. The selection of FCA for processing qualitative information about complex systems lays in the fact that human reasoning -in fact, our Bounded reasoning (BR) skills- about the dynamics and organization of a CS has a qualitative nature. Therefore, human 
reasoning and conjectures about the CS can be expressed in qualitative terms (possibly choosing thresholds and multivalued attributes). Once qualitative hypothesis are presented, even non-symbolic mechanisms for reasoning can be useful to validate the conjectures.

The reasoning process by means of FCA is depicted in Fig. 2 (left). The observer has to select attributes and objects he consider relevant to determine CS dynamics. The global context (with a huge size) is built by means of data extraction and processing, expert observations, data mining, etc. It is expected that reasoning with a contextual selection provide some information about the CS.

The full model consist of events (objects) which have a number of properties (attributes) [3]. They constitute a universal formal context, $\mathbb{M}$ (the monster context). Therefore $\mathbb{M}$ can be considered as the global memory from which subcontexts are extracted. Once a specific subcontext is considered (the contextual selection), it is interesting to consider the knowledge extracted from the formal context (implication basis or association rules [3]). Subcontexts are selected to obtain a feasible reasoning system to predict or analyze the main features of the system [3].

\section{A. A particular application: FCA reasoning for prediction}

Intimately related with the percolation problem, the case of inferring properties about future events is particularly interesting. In this case the evolution of the CS is not predicted by studying new concepts in the lattice, instead, implication basis (and their extension, Luxenburger basis [13]) are used.

The method proposed in [3] consist in predicting the value of selected attributes on future objects. In fact, the process can be understood as the use of implication bases to foresee values (future) for attributes. To relate this method with the study presented in this paper, it is succinctly described (see Fig. 2 left):

- A question on whether a new event (object) has a property (attribute) is raised. Some other properties (attributes) of this object are known $\left\{A_{1}, \ldots A_{n}\right\}$.

- The subcontext induced by a selection of attributes is used to compute a Knowledge Base $\mathcal{L}$, called contextual $K B$. This $\mathrm{KB}$ consists of a set of implications among attributes, extracted from a subcontext.

- A reasoning system is executed on the contextual $\mathrm{KB}$, taking $\left\{A_{1}, \ldots A_{n}\right\}$ as initial facts. The results are attributes inferred from the object.

Note that it only computes those attributes entailed from the set selected by the user. Therefore, it is necessary to understand the topology of the lattice to properly choose the attributes to reason with.

\section{Percolation in CONCEPT LatTices}

In a similar way to the above-depicted application, concept lattices built from a contextual selection can be a sound phenomenological representation when some amount of adequate qualitative knowledge on the CS is collected. To see how robust is the lattice, it is interesting to run experiments focused on the behavior of the lattice when facing new (no previously categorized) elements. In [4] a relationship between the topology of the lattice and the soundness of the reasoning is studied.

Roughly speaking, percolation in concept lattices consists in creating artificial objects and exploring the lattice from the top concept, attempting to fit the intension of the new object with a preexisting concept. The percolation process succeeds when the new object is located in the most specific concept layer of the lattice (see Fig. 2 right).

\section{A. Attributes (and incompatibilities) for the unexpected object}

In the percolation process, an attribute set (the intension of the new object) is randomly generated by selecting, with a certain probability $p$, some of the attributes from $\mathbb{A}$. This process can produce an attribute set containing incompatibilities between attributes. Two attributes are incompatible if they cannot occur at the same time in the domain under study due to its nature (i.e. a person cannot be tall and short at the same time). Since the goal of this experiment is to test the knowledge structure behind the CS against unexpected phenomena, this knowledge structure does not need to be robust (and cannot be) against incompatible attribute sets, as those can never be a representation of a real phenomenon.

Following this idea, in the random generation of objects it is necessary to deal with three different types of features:

Isolated features: Attributes which presents only one possible value, thus cannot suffer of incompatibilities.

Multivalued features: A group of attributes for which only one and always one of the attributes can occur (i.e. \{tall, intermediate, short $\}$ ).

Incompatible feature sets: Sets of attributes that are incompatible to each other, thus only attributes of one of those sets can occur and more than one attribute of the same attribute set can appear at the same time. For example we can consider attribute sets like $\{6,5,4\},\{3,2\}\{1\}$ to refine \{tall, intermediate, short $\}$. When modeling CS, sometimes it is necessary to discretize (by means of thresholds) quantitative features. This situation typically arises when discretizing two or more quantitative features that are incompatible to each other.

\section{PERCOLATION EXPERIMENT}

In this section the phenomenological percolation process is presented and the possible results of the process are explained. The basic idea consist in generating a huge amount of random attribute sets (the intents of potentially unexpected objects) and classifying them, in order to study and measure how deep the generated object is classified. A critical question to analyze is if the object reaches an existing concept or would create a new one.

As in typical percolation phenomena, each percolation action is performed with a certain density factor $p$. In the phenomenological percolation process, the density $p$ is the probability for each attribute $a_{i} \in \mathbb{A}$ to be part of the randomly generated attribute set. That is to say, this probability 
$p$ somehow denotes the density (in number of attributes) of the new object. Therefore for $p=0$ the generated attribute set will be empty and for $p=1$ the attribute set can contain every attribute $a_{i} \in \mathbb{A}$ respecting the restrictions imposed by the declared incompatibilities.

Three kind of attributes have been defined regarding the different incompatibilities they can present. When performing the new object random generation, the density factor $p$ is applied in a different way for each of the three different kind of attributes:

Isolated features: As those cannot suffer of incompatibilities, each of then can be taken with probability $p$.

Multivalued features: As only one but always one of the attributes can occur (due to their nature), the probability $p$ has no effect in this case. Let $K$ be the number of possible attributes for this feature, one of them will be taken with probability $1 / K$.

Incompatible feature sets: In this case there are sets of attributes that are incompatible to each other and only attributes of one of those sets can occur, but more than one attribute of the same set can appear at the same time.

Therefore, first it is necessary to choose one of the attribute sets and then take any number of attributes with probability $p$ from this set, that is:

Let $\left\{A_{1}, \ldots, A_{N}\right\}$ be the incompatible attribute sets, one of these sets $A_{i}$ can be taken with probability:

$$
p\left(A_{i}\right)=\frac{\left|A_{i}\right|}{\sum_{j=0}^{N}\left|A_{j}\right|}
$$

Once an attribute set has been chosen, each of the attributes within the set is taken with probability $p$.

\section{A. Semantic percolation procedure}

The percolation process is performed on a concept lattice extracted from the monster model. The model has been previously obtained by means of data extraction techniques and after performing an attribute selection (lead by the principles of bounded rationality [3]). Also a knowledge base needs to be extracted from the monster context, as it will be necessary to perform the deductive classification on the randomly generated objects. Once the concept lattice and the knowledge base have been obtained, the phenomenological percolation process on it consist of the following steps:

Specify inconsistencies between attributes: This is a previous step that should be performed manually by domain experts. It is necessary to specify the incompatibilities between the attributes of the context, in order to respect the nature of the system under study while generating the new objects. That is, the artificial objects should behave as the real ones.

Percolation action: The act of percolation is the process of generating and classifying the new object, launching it through the concept lattice and measuring how deep it falls. This process should be repeated a number of times for each value

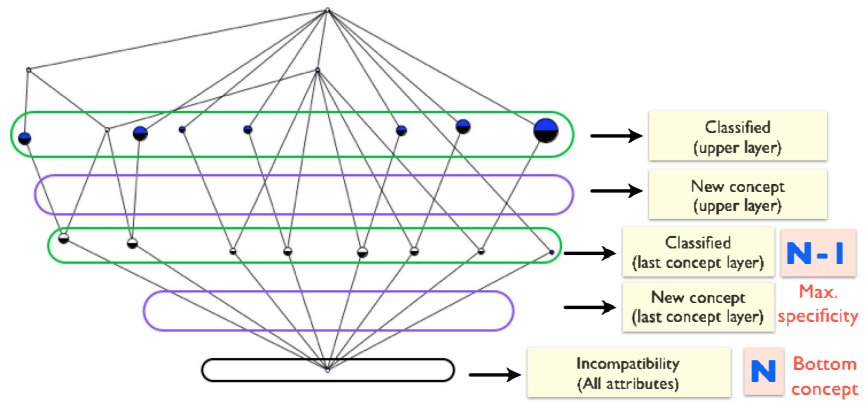

Fig. 3. Percolation levels on the concept lattice

of $p$ in order to compute the mean value, providing a reliable estimate (as it is topically done for random phenomena). Each act of percolation has the following steps:

- Generate the new object (random attribute set) with respect to the density factor $p$ and respecting the specified incompatibilities.

- Classify the new object according to the knowledge base. Attributes are added to the new object in order to complete (augment its intent) the object according with the knowledge base. The added attributes are the entailment of the production system applied on the generated attributes and the knowledge base.

- Finally the object is initialized at the top concept of lattice. The deepness of the place where the concept falls is measured and the kind of concept (new or existing) is stored.

Finally, all results are aggregated, and conclusions on the goodness of the knowledge structure representing the system are extracted. Especially interesting are those critical points considered as phase transitions.

\section{B. Analyzing the percolation}

Since the goal of the percolation process is to test the robustness of the knowledge structure (the concept lattice) representing a complex system, the place where each new generated object falls within the concept lattice after the percolation action should be studied. With this aim, a number of different levels (or degrees) of percolation (see Fig. 3) has been defined (three main levels and two sub-levels) due to their different interpretation:

Existing concept: The object is allocated in a concept.

- The new object falls in a concept within the last layer of concepts (the most specific) of the lattice. This means that the new object has been correctly identified with maximum specificity.

- The new object falls in an upper layer of concepts. That is, the new object is also properly identified but with less specificity.

New concept: The object would create a new concept.

- The new object would create a new concept on the last layer with the most specific concepts of the lattice. This means the lattice lacks of specific enough categories to be able to classify the new object. 
This can occur when the new object has attributes previously incompatible although experts do not claim this fact (that is, it can be possible in CS evolution)

- The new object would generate a new concept on an upper layer of concepts. It can occur when the attribute set lacks of a complete description of features to distinguish any object property.

Incompatibility (Bottom concept): The concept falls down until the bottom concept of the lattice. The bottom concept contains every attribute of $\mathbb{A}$. This means, that in the deductive classification step, the production system has entailed for the new object every possible attribute.

This usually occurs due to incompatibilities in the attribute set (those of the new object) used as initial facts for the production system. It is worthy to note that it is not always possible to detect and avoid every incompatibility between attributes.

Other interesting case occurs when a new object exhibits a new attribute combination that is a refinement of previous most specific concepts. It can be interpreted as that the lattice provides a knowledge organization coarser than the new one.

\section{EXPERIMENTAL RESULTS}

A number experiments have been performed in order to analyze the toughness, against unexpected events, of the knowledge structures obtained from the information available on three study cases of diverse nature:

Spanish soccer league: This is a CS where a great number of levels, factors and agents take part. This dataset has already been used in former works as [3]. The goal for which it was originally created is for simulating human forecasting of soccer matches. The monster context consists of matches (objects) and attributes characterizing the ability of each of the teams involved in the match. These attributes includes different past information about the team on the league (match results, points, goals, etc.) as well as socioeconomic data.

Darfur conflict: This case is an experimental application with a CS where spatial features are relevant, the Darfur conflict, and was also used by the authors in [4]. The conflict represents a very complex system, where information is difficult to be processed and computed, as sources are sparse and noisy.

Baroque artworks tagging: The last study case is the artworks repository Baroque $A r t^{1}$ created by the CulturePlex $\mathrm{Lab}^{2}$. In this case the aim is to analyze the structure and in some cases try to complete and repair the artworks semantic tagging from this repository. This case represents a usual case study in Digital Humanities. This field of research faces cultural complexity, and social representations of knowledge have to be revised and measured. For example, phenomenological aspects of spatial and temporal modeling have had very little importance in the history of qualitative representations. When we expand this discussion to include the spatial and temporal richness of cultural artifacts, the vision of a complex worldview begins to beg for digital means of exposure. In this

\footnotetext{
${ }^{1} \mathrm{http}: / /$ baroqueart.cultureplex.ca/ ${ }^{2} \mathrm{http}: / / \mathrm{www} . c u l t u r e p l e x . c a /$
}
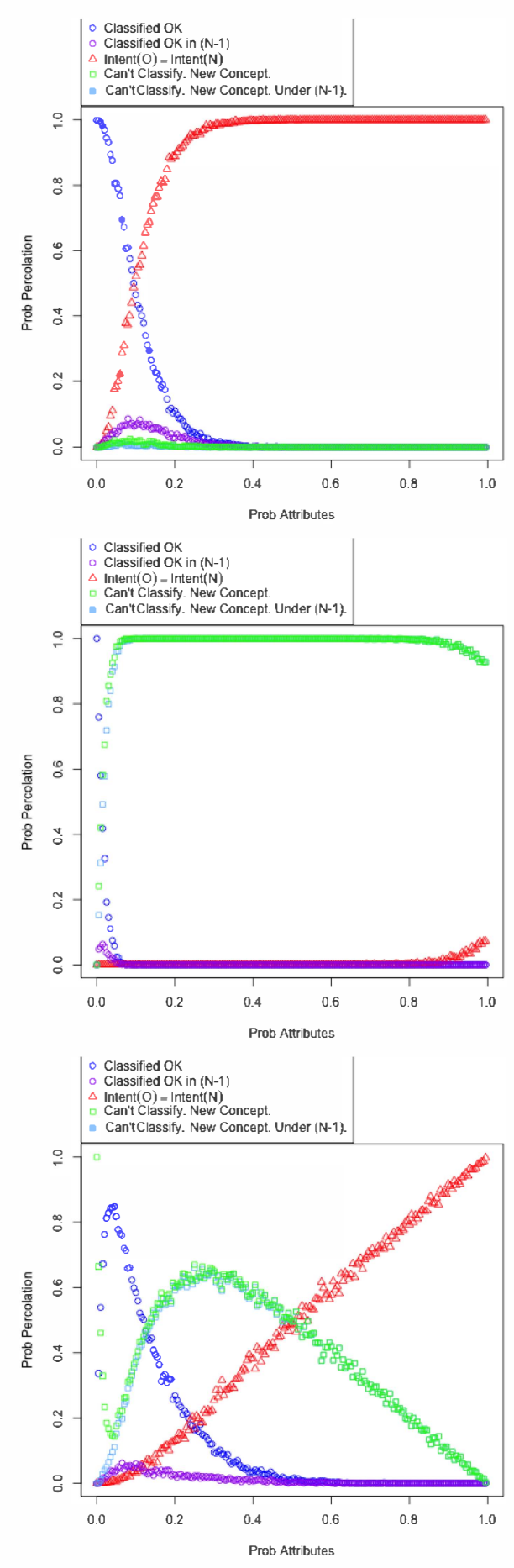

Fig. 4. Experimental results: Spanish soccer league (first), Baroque artworks tagging (second) and Darfur conflict (third).

case, the significance and robustness of representations turns out in a key challenge [10].

For each of these datasets, once the monster context was built and the associated data structures were extracted, a number of percolation experiments have been performed in order to obtain reliable results. The probability $p=[0-1]$ was divided into 200 intervals for which the percolation experiment 
was carried out 1000 times (for each value of $p$ ) and finally a mean value (for these 1000 repetitions) was computed.

Roughly speaking, results show that the methodology depicted in this work provides an useful assessment of both, the robustness of the knowledge structure and the amount of information (instances) populating such knowledge structure. Fig 4 shows results of experiments with the three selected study cases, which presents different features, leading on different behaviors in the percolation experiment. However, results are approximate due to the fact that it is possible to avoid direct incompatibilities but not indirect ones in the attributes random generation. Indirect incompatibilities can lead to a wrong entailment in the production system, although results are quite reasonable:

Spanish soccer league: Results (see Fig. 4, top) show that for very low values of $p$ the concept lattice is able to identify (classify) everything (blue circles), that is, no unexpected objects (new concept) appear (green squares). If $p$ is increased, the number of incompatibilities (red triangles) raises significantly and the number of correctly identified objects decreases, until $p=0.3$ approx. where it becomes practically 0 .

The interpretation of this behavior is that the associated concept lattice and knowledge base are robust against unexpected objects as the randomly generated new objects are perfectly identified by the production system or declared as inconsistent, but none declared as unknown.

Therefore the results of this experiment shows that this dataset presents both, a good representation and objects population on the domain, which agrees with our experiences while applying reasoning techniques on the data.

Baroque artworks tagging: In this case study, results (see Fig. 4, center) show that when $p$ is almost zero, there are few well identified (classified) objects (blue circles) and for the rest values of $p$ the knowledge base can not properly identify unexpected objects (green squares). Finally when $p$ is close to one, some incompatibilities (red triangles) appears.

The interpretation of this behavior can be that the associated concept lattice structure is consistent with the domain it represents but the information it contains is quite poor, that is, it is necessary to collect more information on the domain in order to fill the gaps. Moreover these conclusions agree with the experience we have after working with this dataset.

Darfur conflict: Results (see Fig. 4 bottom) show a mixture of behaviors for different values of $p$. It presents the three kind of possible results in this percolation experiment, well-classified (blue circles), unrecognized (red green squares) object (new concept), and incompatibility (red triangles).

The fact that the last two phenomena occur at the same time seems to be somehow chaotic. And in fact this chaotic behavior agrees with the experience we have while applying reasoning techniques on the dataset, as the entailed results were chaotic as well. This dataset is noisy and full of inconsistencies, thus, the representation it provides is not reliable.

\section{CONCLUDING REMARKS AND FUTURE WORK}

The concept of phenomenological percolation, as a FCAbased metaphor of the management of new (unexpected) objects within semantic networks, has been studied from the point of view of qualitative reasoning on CS. Results show that concept lattices providing a sound representation of features on CS have specific structures. This conclusion is similar to the relationship between the topological structure of the lattice and its soundness as a good reconstruction of the CS [4].

Future work will focus on the algebraic and topological nature of the change. Concept lattices strongly depends on the observation set, and it was shown that in some cases abundance of information worsens the quality of the reasoning [4]. When a number of unexpected objects are observed, BR techniques have to reevaluate both, selection and reasoning with attributes.

Acknowledgments Supported by TIC-6064 project, of Junta de Andalucía co-financed by FEDER funds; and TIN2013-41086-P project of Spanish Ministry of Science and Innovation.

\section{REFERENCES}

[1] Aranda-Corral, G. A., Borrego-Díaz, J., Galán-Páez, J.: Simulating Language Dynamics by means of Concept Reasoning. To appear in Lecture Notes of the Inst for Comput. Sci,, Social Informatics and Telecom. Eng. vol. 134. Springer (2014)

[2] Aranda-Corral, G. A., Borrego-Díaz, J., Galán-Páez, J.: Qualitative Reasoning on Complex Systems from Observations. Proc. Int. Conf. on Hybrid Artificial Intelligence Systems 2013, LNCS vol. 8073, pp.202211, Springer, Heidelberg (2013).

[3] Aranda-Corral, G. A., Borrego-Díaz, J., Galán-Páez, J.: Complex Concept Lattices for Simulating Human Prediction in Sport, Journal of Systems Science and Complexity, 26 (1), pp. 117-136, (2013).

[4] G.A. Aranda-Corral, J. Borrego-Díaz, J. Galán-Páez, On the Phenomenological Reconstruction of Complex Systems-The Scale-Free Conceptualization Hypothesis, Systems Research and Behavioral Science 30(6): 716-734 (2013).

[5] F. Bagnoli, Interaction Based Computing in Physics, in Computational Complexity Theory, Techniques, and Applications (R. A. Meyers ed.) pp 1644-1662 Springer (2012).

[6] L.W. Barsalou, Situated Conceptualization, chap. 18 in Handbook of Categorization in Cognitive Science (H. Cohen and C. Lefebvre eds.). Elsevier (2005).

[7] Beraldo D., Galán-Paez J.G. (2013). The \#OCCUPY network on Twitter and the challenges to social movements theory and research, International Journal of Electronic Governance, 6 (4) 319.

[8] Borrego-Díaz, J., Galán-Páez, J., Discovering New Sentiments from the Social Web. To appear in Proc. Collective Intelligence Conference. MIT, 2014

[9] Bourgine, P., Chavalarias, D., Perrier, E. (eds.): The CSS Roadmap for the Science of Complex Systems. (2009).

http://www.assystcomplexity.eu/db/assyst/ASSYST_roadmap2009_2. pdf

[10] J. Drucker, Humanistic Theory and Digital Scholarship in Debates in the Digital Humanities (M.H. Gold ed.), pp. 85-95 U. Minessota Press (2012).

[11] Ganter, B., Wille, R.: Formal Concept Analysis. Mathematical Foundations. Springer, (1999).

[12] Guigues, J.-L., Duquenne, V.: Familles minimales d' implications informatives resultant d'un tableau de donnees binaires. Math. Sci. Humaines 95, pp. 5-18. (1986).

[13] Luxenburger, M.: Implications partielles dans un contexte. Math. Inf. Sci. hum. 113 pp. 35-55. (1991).

[14] M. San-Miguel, JH Johnson, J Kertesz, K Kaski, A Díaz-Guilera, RS.MacKay, V Loreto, P. Érdi, D Helbing: Challenges in Complex Systems Science. Eur. Phys. J. Special Topics 214, 245-271 (2012)

[15] Vigo, R. Complexity over Uncertainty in Generalized Representational Information Theory (GRIT): A Structure-Sensitive General Theory of Information. Information 2013, 4, 1-30. 